\title{
HYBRID FREQUENCY-TIME DOMAIN METHOD FOR THREE-DIMENSIONAL SEISMIC ANALYSES OF NONLINEAR SOILS
}

\author{
Francesca Taddei $^{1}$, Thi Hoa Nguyen ${ }^{1}$, and Gerhard Müller ${ }^{1}$ \\ ${ }^{1}$ Chair of Structural Mechanics,Technical University of Munich \\ Arcisstr. 21, 80333 Munich \\ e-mail: \{francesca.taddei, hoa.nguyen, gerhard.mueller\}@tum.de
}

Keywords: Soil-Structure Interaction (SSI), Wave Propagation Theory, Hybrid frequency-time domain (HFTD), Nonlinear Soil, 3D Effects, Integral Transform Method (ITM)

\begin{abstract}
The simulation of the behavior of soil-structure interaction (SSI) systems subjected to seismic waves can be conveniently carried out in the frequency domain, to account for the frequency-dependent properties of the infinite soil. However, the soil exhibits nonlinear behavior even at small values of the strain level, so that its shear modulus and damping ratio vary as a functions of the strain. It is important to consider the strain-and frequency-dependent properties of the soil in a seismic SSI simulation. Since frequency domain (FD) analyses are not suitable for nonlinear systems, time domain (TD) integration algorithms are commonly adopted. An accurate way of including the frequency-dependent soil into a transient analysis is to use convolution integrals, which requires linearity of the system and leads to a high computational cost. Hybrid frequency-time domain (HFTD) methods represent an efficient alternative. Most of the existing HFTD methods idealize the soil behavior according to the one-dimensional (1D) wave propagation theory, which is a major simplification of the complex three dimensional (3D) theory.

In this contributions we present a HFTD method, where the soil is described as a $3 D$ continuum governed by Lamé's equation of elastodynamics and the problem is solved with the integral transform method (ITM). The shear modulus and the damping of the soil are nonlinear functions of the strain level. The seismic waves (the loading) can be represented by spatial waves with an arbitrary distribution in time and space. The response of the soil to the seismic waves is first solved in the FD assuming a linearized elastic soil subjected to input waves, the response is transformed into the TD and the maximum strain is evaluated at different depths. According to the maximum strain, the shear modulus and the damping at different depths is updated and the process is iterated until convergence. The proposed method is compared to the reference $1 D$ nonlinear method and it is applied for a multi-dimensional analysis of a nonlinear soil. The limitations of the method are also described.
\end{abstract}




\section{INTRODUCTION}

Structures and the underlying soil can exhibit nonlinear behavior during strong earthquakes. Nonlinearities can arise in the structure, in the soil, or/and at the interface between structure and soil (i.e. base isolation, uplift). In order to simulate the nonlinear interaction between the soil and the structure during a seismic event, transient analyses in the time domain are necessary. It is common to use discretized numerical models for simulating structural problems in civil engineering; in particular, the finite element method (FEM) is the most established approach, thanks to its suitability for nonlinear and anisotropic materials.

However, the FEM fails to represent the real endless dimension of the soil because the energy cannot be transported to infinity. A possible solution is to enlarge the soil domain and move its boundary away from the structure at a sufficient distance. This leads to a model with a large number of elements, for which the run time becomes prohibitive.

Another complex aspect of nonlinear seismic analysis is the correct estimation of the seismic loads, which do not act directly on the structures but result from the spatial and temporal variation of the seismic displacement field. The latter, in turn, can be influenced by the foundation and the structure. This aspect must be taken into account when selecting the analysis method.

In the past decades, much effort has been made in order to find valid alternatives to the classical FEM representation for nonlinear soil-structure interaction (SSI) problems. The different methods can be classified into two main groups: direct methods and substructure methods. In both cases the aim is to overcome the difficulty of modeling the unbounded domain of the soil, accounting for the radiation conditions at infinity and the nonlinearities of the system due to seismic events.

In the direct methods, the structure and part of the soil near the foundation (near field) are modeled using the same technique, usually the FEM. In order to satisfy the radiation condition, mathematical artifacts at the boundary of the soil's near field must be added. These are called transmitting boundaries (TB) and are local approximations of boundary conditions (local in time and space) and their coefficients are frequency-independent. Direct methods can account for nonlinearities in the structure as well as in the soil's near field and can be solved using direct time integration methods such as central difference method or Newark method. The soil's far field is assumed to remain linear. Applications of direct methods can be found in [8]. The direct method for nonlinear SSI system is computationally demanding.

The substructure methods involve the application of different techniques for different parts of the systems and their coupling at the interaction surfaces. Usually the FEM is used for the structure and a different numerical method is used for the soil. If nonlinearities occur, the soil model must be defined in the TD and this would involve convolution integrals. In this case, the SSI boundary conditions are global in time and space, as they require the information of all the time steps from the beginning and all the degrees of freedom (DoF) at the substructure interface. Substructure methods can account for nonlinearities in the structure as well as at the soil-structure interface, whereas the soil is assumed to remain linear. A classical reference for the theory of substructure methods is Wolf [23]. An example of application can be found in [18].

Alternatives to transient analysis are the hybrid time-frequency domain (HTFD) approaches, where the system of equations is solved iteratively transforming back and forth from the TD to the FD and vice versa. The existing HFTD methods assume the soil approximated as a onedimensional system (either as a compressional bar for P-waves or as a shear beam for S-waves).

In this contribution we present an extension of the HTFD, where the three-dimensionality 


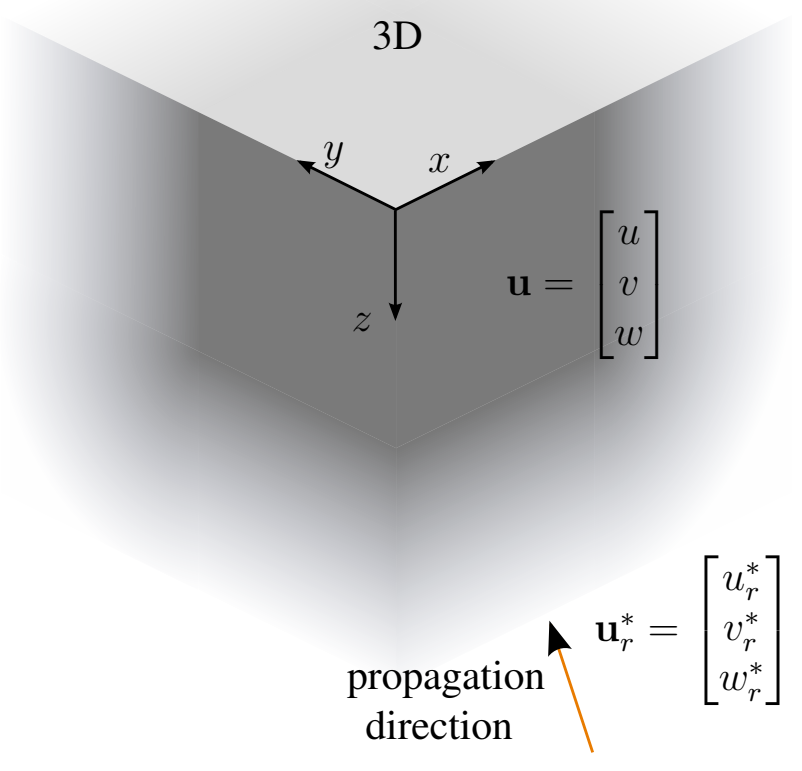

a)
$1 \mathrm{D}$

P-waves

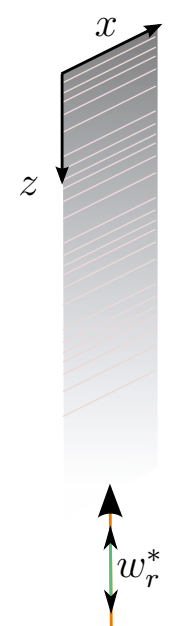

b)
$1 \mathrm{D}$

SH-waves

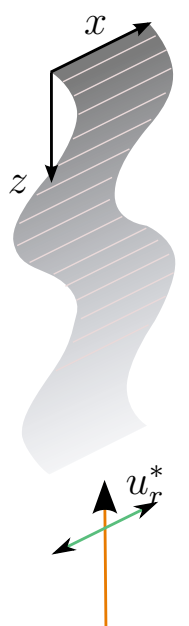

c)

Figure 1: a) 3D free field, b) 1D representation of the free field subjected to P-waves, c) 1D representation of the free field subjected to $\mathrm{S}$-waves.

of the problem can be account for and the solution is found iteratively using both time- and frequency-domain results. The focus in on the nonlinear response of the free field and the equations are derived only for the nonlinear soil. However, a coupling to a nonlinear structure using transfer functions is straightforward.

\section{THREE-DIMENSIONAL PROPAGATION OF SEISMIC WAVES}

Let us first analyze a linear isotropic soil. The propagation of seismic waves through the soil can be described using the theory of elasticity applied to a three dimensional homogenous half space. Different forms of waves (shear, compressional and Rayleigh waves) can propagate simultaneously, generating a complex three dimensional displacement field $\mathbf{u}$. In seismic analysis it is of interest 1) how the soil amplifies or de-amplifies the signal as the waves propagate through the medium towards the structure, and 2) how its flexibility influences the overall dynamic response of the structure. For both aspects, the extent of the effects depends on the properties of the soil and on the frequency content and spatial distribution of the seismic excitation. The propagation of seismic waves through a non-homogenous half space is even more complicated, because of additional Stoneley waves, Love waves and evanescent modes.

The problem is shown in Fig. 17a and can be described with the Lamé equation in index notation:

$$
\left.G u^{i}\right|_{j} ^{j}+\left.(\lambda+G) u^{j}\right|_{j} ^{i}-\rho \ddot{u}^{i}=0 \quad \text { with } \quad i, j=x, y, z
$$

where $u^{x}=u, u^{y}=v$ and $u^{z}=w$ are the componenet of the displacement vector $\mathbf{u}, \rho$ is the soil mass density, $\lambda$ and $G$ are the Lamé-constant and can be estimated from the Youngs's modulus 
$E$ and Poisson's ratio $\nu$ :

$$
G=\frac{E}{2(1+\nu)} \quad \text { and } \quad \lambda=\frac{\nu E}{(1+\nu)(1-2 \nu)} .
$$

The Lamé equation, Eq. (1), is defined in the original space $(x, y, z, t)$ and is valid for a linear elastic isotropic and homogeneous continuum. Using the Helmholtz decomposition and a threefold Fourier transformation one obtains a system of four decoupled ordinary differential equations which can be solved with an exponential approach in the image space $\left(k_{x}, k_{y}, z, \omega\right)$ [4]. This approach is referred to as integral transform method (ITM). The solution expressed in terms of potentials $\bar{\phi}$ and $\bar{\psi}_{i}$ reads:

$$
\begin{gathered}
\bar{\phi}\left(k_{x}, k_{y}, z, \omega\right)=A_{1} e^{\lambda_{1} z}+A_{2} e^{-\lambda_{1} z} \\
\bar{\psi}_{i}\left(k_{x}, k_{y}, z, \omega\right)=B_{i 1} e^{\lambda_{2} z}+B_{i 2} e^{-\lambda_{2} z} \quad \text { with } \quad i=x, y, z \\
\text { with } \quad \lambda_{1}=-i \sqrt{\frac{\omega^{2}}{c_{p}^{2}}-k_{x}^{2}-k_{y}^{2}}, \\
\lambda_{2}=-i \sqrt{\frac{\omega^{2}}{c_{s}^{2}}-k_{x}^{2}-k_{y}^{2}}, \\
k_{p}=\frac{\omega}{c_{p}} \text { and } k_{s}=\frac{\omega}{c_{s}}
\end{gathered}
$$

where $c_{p}$ and $c_{s}$ are the compressional and shear wave velocity respectively and $k_{x}$ and $k_{y}$ are the horizontal wave numbers. One can demonstrate that the potential $\bar{\psi}_{z}$ can be set to zero, still maintaining the complete correct 3D solution [6]. The displacements in the image space can be obtained as:

$$
\overline{\mathbf{u}}=\left(\begin{array}{c}
\bar{u} \\
\bar{v} \\
\bar{w}
\end{array}\right)=\underbrace{\left[\begin{array}{cccccc}
i k_{x} & i k_{x} & 0 & 0 & -\lambda_{2} & \lambda_{2} \\
i k_{y} & i k_{y} & \lambda_{2} & -\lambda_{2} & 0 & 0 \\
\lambda_{1} & -\lambda_{1} & -i k_{y} & -i k_{y} & i k_{x} & i k_{x}
\end{array}\right]}_{\boldsymbol{K}_{\overline{\mathbf{u}}}} \underbrace{\left(\begin{array}{c}
A_{1} e^{\lambda_{1} z} \\
A_{2} e^{-\lambda_{1} z} \\
B_{x 1} e^{\lambda_{2} z} \\
B_{x 2} e^{-\lambda_{2} z} \\
B_{y 1} e^{\lambda_{2} z} \\
B_{y 2} e^{-\lambda_{2} z}
\end{array}\right)}_{c}
$$

where the vector $c$ contains the 6 unknown coefficients of the solution. The stresses can be estimated and expressed as:

$$
\left(\begin{array}{c}
\bar{\sigma}_{x} \\
\bar{\sigma}_{y} \\
\bar{\sigma}_{z} \\
\bar{\sigma}_{x y} \\
\bar{\sigma}_{y z} \\
\bar{\sigma}_{z x}
\end{array}\right)=G \underbrace{\left[\begin{array}{cccccc}
-\left(2 k_{x}^{2}+\frac{\lambda}{G} k_{p}^{2}\right) & -\left(2 k_{x}^{2}+\frac{\lambda}{G} k_{p}^{2}\right) & 0 & 0 & -2 i k_{x} \lambda_{2} & 2 i k_{x} \lambda_{2} \\
-\left(2 k_{y}^{2}+\frac{\lambda}{G} k_{p}^{2}\right) & -\left(2 k_{y}^{2}+\frac{\lambda}{G} k_{p}^{2}\right) & 2 i k_{y} \lambda_{2} & -2 i k_{y} \lambda_{2} & 0 & 0 \\
2 k_{r}^{2}-k_{s}^{2} & 2 k_{r}^{2}-k_{s}^{2} & -2 i k_{y} \lambda_{2} & 2 i k_{y} \lambda_{2} & 2 i k_{x} \lambda_{2} & -2 i k_{x} \lambda_{2} \\
-2 k_{x} k_{y} & -2 k_{x} k_{y} & i k_{x} \lambda_{2} & -i k_{x} \lambda_{2} & -i k_{y} \lambda_{2} & i k_{y} \lambda_{2} \\
2 i k_{y} \lambda_{1} & -2 i k_{y} \lambda_{1} & \lambda_{2}^{2}+k_{y}^{2} & \lambda_{2}^{2}+k_{y}^{2} & -k_{x} k_{y} & -k_{x} k_{y} \\
2 i k_{x} \lambda_{1} & -2 i k_{x} \lambda_{1} & k_{x} k_{y} & k_{x} k_{y} & -\left(\lambda_{2}^{2}+k_{x}^{2}\right) & -\left(\lambda_{2}^{2}+k_{x}^{2}\right)
\end{array}\right]}_{\boldsymbol{K}_{\bar{\sigma}}} \boldsymbol{c}
$$

with

$$
k_{r}^{2}=k_{x}^{2}+k_{y}^{2}
$$


In short notation, we can write:

$$
\begin{aligned}
& \overline{\mathbf{u}}=\boldsymbol{K}_{\overline{\mathbf{u}}} \boldsymbol{c}, \\
& \overline{\boldsymbol{\sigma}}=\boldsymbol{K}_{\bar{\sigma}} \boldsymbol{c} .
\end{aligned}
$$

The two matrices $\boldsymbol{K}_{\overline{\mathbf{u}}}$ and $\boldsymbol{K}_{\bar{\sigma}}$ depend on the excitation circular frequency $\omega$, the soil material and the horizontal wave numbers $k_{x}$ and $k_{y}$. Based on given boundary conditions at certain $z$ coordinates the unknown coefficients vector $c$ can be found.

The application of the the material law delivers the strain components in the original space :

$$
\begin{aligned}
\sigma_{x} & =\lambda \widehat{\varepsilon}+2 G \varepsilon_{x} & \tau_{x y} & =\tau_{y x}=G \gamma_{x y} \\
\sigma_{y} & =\lambda \widehat{\varepsilon}+2 G \varepsilon_{y} & \tau_{y z} & =\tau_{z y}=G \gamma_{y z} \\
\sigma_{z} & =\lambda \widehat{\varepsilon}+2 G \varepsilon_{z} & \tau_{z x} & =\tau_{x z}=G \gamma_{z x}
\end{aligned}
$$

with

$$
\widehat{\varepsilon}=\varepsilon_{x}+\varepsilon_{y}+\varepsilon_{z}
$$

Adding $\sigma_{x}, \sigma_{y}$ and $\sigma_{z}, \widehat{\varepsilon}$ is estimated as:

$$
\widehat{\varepsilon}=\frac{\sigma_{x}+\sigma_{y}+\sigma_{z}}{3 \lambda+2 G}
$$

The strain components $\varepsilon_{x}, \varepsilon_{y}$ and $\varepsilon_{z}$ result from inserting Eq. (11) into Eq. (9). The three shear strain components $\gamma_{x y}, \gamma_{y z}$ and $\gamma_{z x}$ can be estimated directly from the respective shear stress through division by the shear modulus $G$. Thus, a matrix formulation of the stress-strain relationship reads:

$$
\underbrace{\left(\begin{array}{c}
\varepsilon_{x} \\
\varepsilon_{y} \\
\varepsilon_{z} \\
\gamma_{x y} \\
\gamma_{y z} \\
\gamma_{z x}
\end{array}\right)}_{\varepsilon}=\underbrace{\left[\begin{array}{cccccc}
\frac{1}{2 G}\left(1-\frac{\lambda}{3 \lambda+2 G}\right) & -\frac{1}{2 G} \frac{\lambda}{3 \lambda+2 G} & -\frac{1}{2 G} \frac{\lambda}{3 \lambda+2 G} & 0 & 0 & 0 \\
-\frac{1}{2 G} \frac{\lambda}{3 \lambda+2 G} & \frac{1}{2 G}\left(1-\frac{\lambda}{3 \lambda+2 G}\right) & -\frac{1}{2 G} \frac{\lambda}{3 \lambda+2 G} & 0 & 0 & 0 \\
-\frac{1}{2 G} \frac{\lambda}{3 \lambda+2 G} & -\frac{1}{2 G} \frac{\lambda}{3 \lambda+2 G} & \frac{1}{2 G}\left(1-\frac{\lambda}{3 \lambda+2 G}\right) & 0 & 0 & 0 \\
0 & 0 & 0 & \frac{1}{G} & 0 & 0 \\
0 & 0 & 0 & 0 & \frac{1}{G} & 0 \\
0 & 0 & 0 & 0 & \frac{1}{G}
\end{array}\right]}_{\boldsymbol{K}_{\varepsilon \sigma}=\boldsymbol{K}_{\bar{\varepsilon} \bar{\sigma}}} \underbrace{\left(\begin{array}{c}
\sigma_{x} \\
\sigma_{y} \\
\sigma_{z} \\
\sigma_{x y} \\
\sigma_{y z}
\end{array}\right)}_{\boldsymbol{\sigma}}
$$

The assumption of linearized elastic material allows the application of the material law also in the image space. Thus,

$$
\begin{gathered}
\boldsymbol{K}_{\varepsilon \sigma}=\boldsymbol{K}_{\bar{\varepsilon} \bar{\sigma}} \quad \text { and } \\
\bar{\varepsilon}=\boldsymbol{K}_{\bar{\varepsilon} \bar{\sigma}} \bar{\sigma} \quad .
\end{gathered}
$$

Combining Eq. 8 and Eq. 13 , the relationship between the strains $\bar{\varepsilon}$ and the vector of the unknowns coefficients $c$ is established:

$$
\bar{\varepsilon}=\underbrace{K_{\bar{\varepsilon} \bar{\sigma}} K_{\bar{\sigma}}}_{K_{\bar{\varepsilon}}} c
$$

For a layered half space with $N$ isotropic layers, the system of equations is obtained by overlapping the matrices of the adjacent layers and by enforcing continuity and equilibrium 


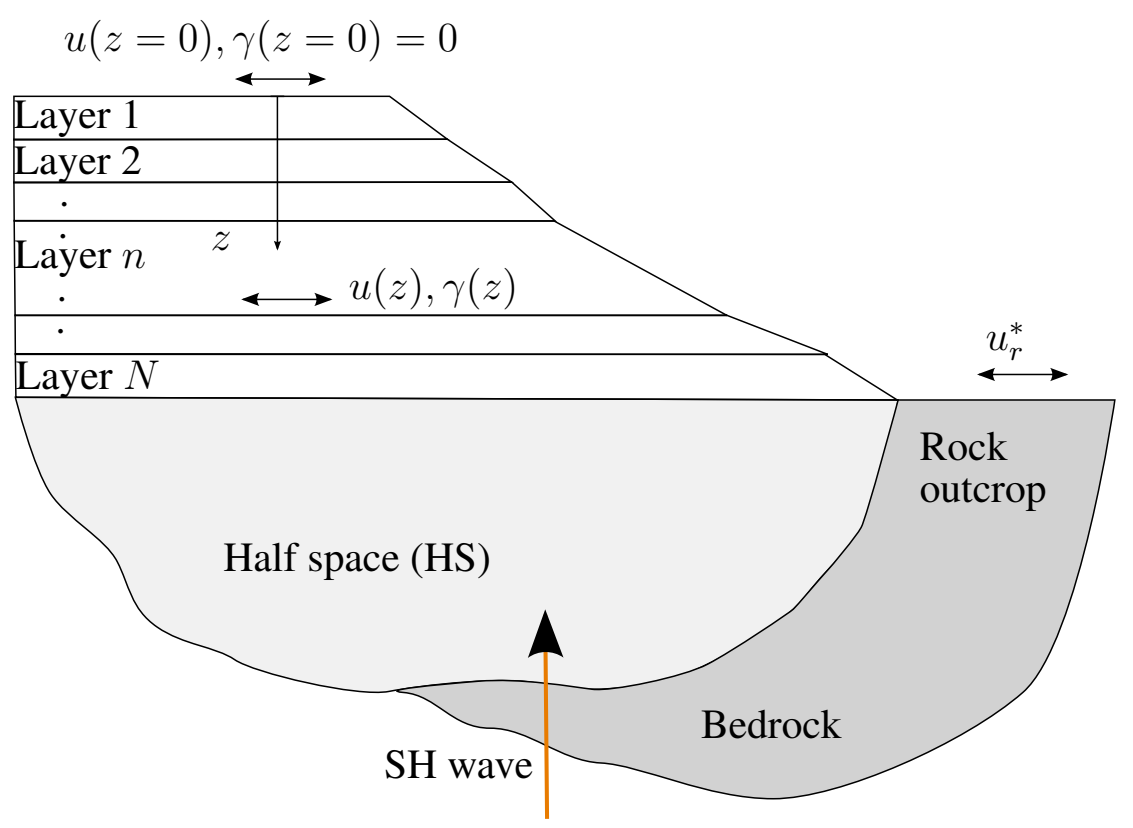

Figure 2: One-dimensional representation of the load application for a seismic analysis of the free field.

at their interfaces. If at the base of the layers there is a bedrock, a fixed boundary condition is enforced at the base and the system has $6 N$ unknowns. If the layers are underlain by an homogenous half space, the radiation condition must be satisfied and the system has $6(N-1)+3$ unknowns.

The unknowns are found with the additional boundary conditions concerning the load. For a free field (soil without a coupled structure) subjected to a seismic excitation, one of the boundary conditions is the zero-stress condition at the surface. A further boundary condition is the equilibrium of stresses at the location where the seismic excitation is applied, which leads to a condition between stresses and displacements at that location and therefore to the remaining unknowns of the problem.

The definition of the seismic input and its location depends on the available information about the seismic event and on the aim of the analysis. In this contribution, according to Kausel [11], we assume that the seismic input is known in form of a motion $\mathbf{u}_{r}^{*}$ at the rock outcrop, which is the surface of the rock without taking into consideration the layers (see Fig. 2).

If at the base of the layers there is a bedrock, $\mathbf{u}_{r}^{*}$ can be directly used as a root-point-excitation condition, after transformation into the image space. If the layers are underlain by an homogenous half space, equivalent seismic stresses are computed according to Kausel [11] and applied at the interface between half space and layers.

Once the problem is solved in the image space, the solution is transformed back into the original space for further calculations (see Fig. 3). In the following paragraphs, ... indicates the quantity in the image space.

\subsection{Three-Dimensional Transfer Functions for the Free Field}

Using the ITM, it is possible to express the relationship between various response parameters of the soil, such as displacements, velocities, accelerations, stresses and strains, and an input motion parameter such as the bedrock displacement vector $\mathbf{u}_{r}^{*}$. This relationship is referred

\footnotetext{
${ }^{1}$ Pages 356-357
} 


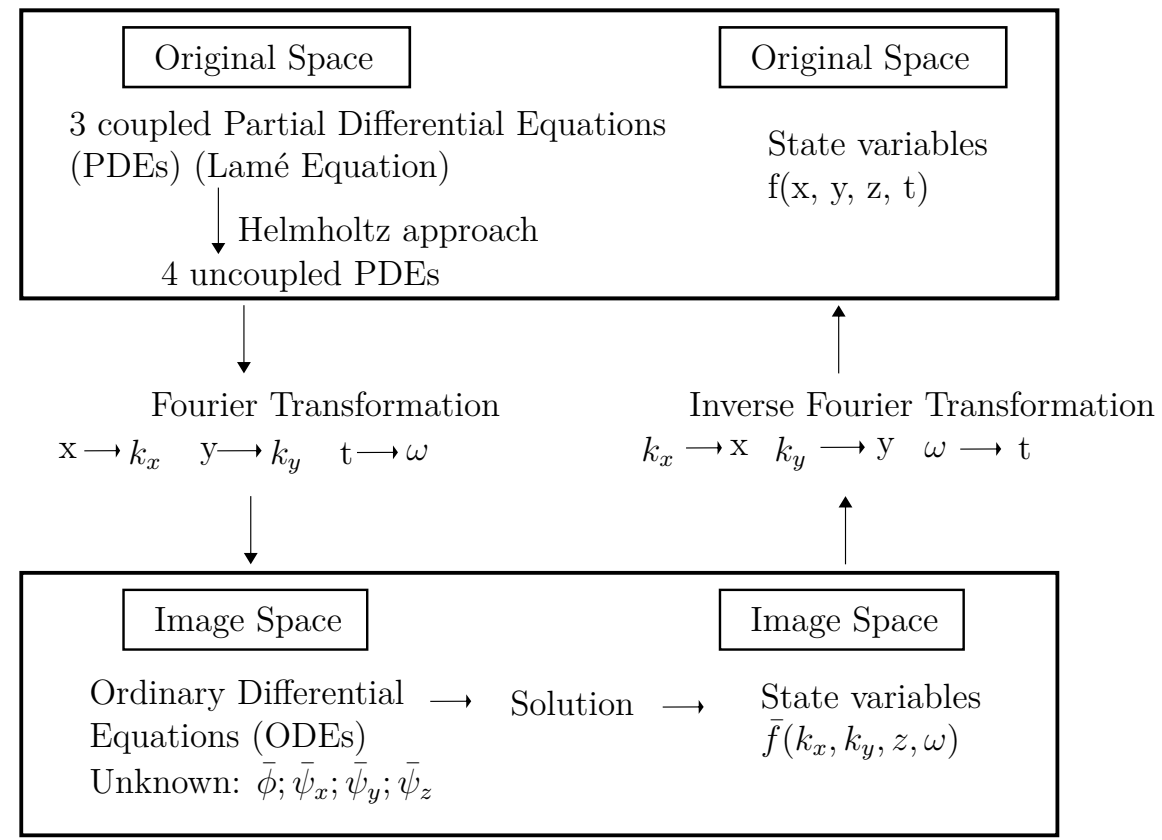

Figure 3: Integral transform method for the solution of the Lamé equations.

to as transfer function. This paragraph explains how to obtain the three-dimensional transfer functions for the displacements and the formulation is shown for a single stratum with thickness $h$ subjected to input motions, to maintain clarity. The same procedure can be applied to any arbitrary horizontally layered soil over a bedrock or an elastic half space.

For a stratum subjected to input motions at the bedrock level, the boundary conditions are:

- BC 1: zero stresses at the soil surface

$\rightarrow\left[\bar{\sigma}_{z}\left(k_{x}, k_{y}, z=0, \omega\right), \bar{\sigma}_{y z}\left(k_{x}, k_{y}, z=0, \omega\right), \bar{\sigma}_{z x}\left(k_{x}, k_{y}, z=0, \omega\right)\right]^{T}=[0,0,0]^{T}$,

- BC 2: the displacements at the interface bewteen bedrock and stratum are given $\rightarrow\left[\bar{u}\left(k_{x}, k_{y}, z=h, \omega\right), \bar{v}\left(k_{x}, k_{y}, z=h, \omega\right), \bar{w}\left(k_{x}, k_{y}, z=h, \omega\right)\right]^{T}=\left[\bar{u}_{r}^{*}, \bar{v}_{r}^{*}, \bar{w}_{r}^{*}\right]^{T}$,

which lead to the following expression:

$$
\left.\left.\left(\begin{array}{c}
A_{1} \\
A_{2} \\
B_{x 1} \\
B_{x 2} \\
B_{y 1} \\
B_{y 2}
\end{array}\right)=[\underbrace{G\left[\begin{array}{cccccc}
2 k_{r}^{2}-k_{s}^{2} & 2 k_{r}^{2}-k_{s}^{2} & -2 i k_{y} \lambda_{2} & 2 i k_{y} \lambda_{2} & 2 i k_{x} \lambda_{2} & -2 i k_{x} \lambda_{2} \\
2 i k_{y} \lambda_{1} & -2 i k_{y} \lambda_{1} & \lambda_{2}^{2}+k_{y}^{2} & \lambda_{2}^{2}+k_{y}^{2} & -k_{x} k_{y} & -k_{x} k_{y} \\
2 i k_{x} \lambda_{1} & -2 i k_{x} \lambda_{1} & k_{x} k_{y} & k_{x} k_{y} & -\left(\lambda_{2}^{2}+k_{x}^{2}\right) & -\left(\lambda_{2}^{2}+k_{x}^{2}\right) \\
i k_{x} e^{\lambda_{1} h} & i k_{x} e^{-\lambda_{1} h} & 0 & 0 & -\lambda_{2} e^{\lambda_{2} h} & \lambda_{2} e^{-\lambda_{2} h} \\
i k_{y} e^{\lambda_{1} h} & i k_{y} e^{-\lambda_{1} h} & \lambda_{2} e^{\lambda_{2} h} & -\lambda_{2} e^{-\lambda_{2} h} & 0 & 0 \\
\lambda_{1} e^{\lambda_{1} z} & -\lambda_{1} e^{-\lambda_{1} h} & -i k_{y} e^{\lambda_{2} h} & -i k_{y} e^{-\lambda_{2} h} & i k_{x} e^{\lambda_{2} h} & i k_{x} e^{-\lambda_{2} h}
\end{array}\right]}_{K_{B C}}]^{-1}\right] \quad \begin{array}{c}
0 \\
0 \\
0 \\
\bar{u}_{r}^{*} \\
\bar{v}_{r}^{*} \\
\bar{w}_{r}^{*}
\end{array}\right)
$$

The coefficients $A_{1}, A_{2}, B_{x 1}, B_{x 2}, B_{y 1}, B_{y 2}$ are then introduced in Eq. 8 to find the displacements and stresses in the image space. Finally, the inverse Fourier transformation leads to the spatial and temporal distribution of the ground motions due to the input motions $\mathbf{u}_{r}^{*}$. Because both input $\mathbf{u}_{r}^{*}$ and output $\mathbf{u}$ have three components and each component depends on the position in space and time, it is not possible to establish a simple definition for the three-dimensional transfer functions, which usually relate scalar values. 
However, for the method proposed in this contribution, it is necessary to choose a representative value of the strain vector for each horizontal plane in the middle of each layer, which will be used in the nonlinear material model. This can be done either observing a specific point of horizontal coordinate $\widehat{x}$ and $\widehat{y}$ or using a spatial averaging method to estimate a representative value.

In order to overcome this difficulty, in most studies on free field response analysis, a major assumption is commonly adopted: the seismic input was generated far enough from the area of interest, so that the wavefront can be considered plane and all the point in the horizontal plane moves in phase and experience the same motion. In the image space, this condition is represented by a function which is equal to zero everywhere except for $k_{x}=k_{y}=0$. In this special case, Eq. 5 and Eq. 15 shows that $\bar{u}$ only depends on $A_{1}$ and $A_{2}$ (related to $\bar{\Phi}$, P-waves), which in turn only depend on $u_{r}^{*}$, so that a straightforward definition for the transfer function $u / u_{r}^{*}$ is possible. Analogously, this apply for $v / v_{r}^{*}$ and $w / w_{r}^{*}$. This means that for $k_{x}=k_{y}=0$, the three dimensional problem can be treated as a one dimensional problem, where each component of the displacement vector can be treated independently.

\subsection{One-Dimensional Transfer Functions for the Free Field}

Under the assumption of a plane horizontal wavefront $\left(k_{x}=k_{y}=0\right)$, the problem in $x$-direction is uncoupled from the problem in $y$-direction and the axial and shear motions do not affect each other. Fig. 10 and Fig. 1p shows that, in 1D, P-waves and SH-waves can be treated separately and the vertical and horizontal transfer functions for a layered soil, $w(z) / w_{r}^{*}$ and $u(z) / u_{r}^{*}$ respectively, can be computed independently at different soil depths. The transfer functions are defined in frequency domain and take into account the frequency-dependent steady-state response of the 1D free field.

We consider a soil made up of $N$ layers with thickness $h_{n}$, shear wave velocity $c_{s, n}$, shear modulus $G_{n}$ and density $\rho_{n}$ underlain by a homogenous half space, subjected to a SH-wave. The transfer functions $\boldsymbol{F}_{1}$ for the displacements, evaluated at the top of each layer, are obtained using the stiffness matrix of the soil $\boldsymbol{K}$ :

$$
\boldsymbol{F}_{1}(\omega)=\left(\begin{array}{c}
u_{1} / u_{r}^{*} \\
u_{2} / u_{r}^{*} \\
u_{3} / u_{r}^{*} \\
\cdots \\
u_{n} / u_{r}^{*} \\
u_{r} / u_{r}^{*}
\end{array}\right)=\boldsymbol{u}_{1 D} / u_{r}^{*}=\boldsymbol{K}^{-1}\left(\begin{array}{c}
0 \\
0 \\
0 \\
\ldots \\
0 \\
K_{H S}
\end{array}\right)
$$

where $\boldsymbol{K}$ is the stiffness matrix of the layered soil and $K_{H S}=i \omega \rho_{H S} c_{s, H S}$ is the stiffness of the underlying half space. The dependency on the circular frequency $\omega$ is omitted for clarity.

This transfer matrix can be applied also to estimate the velocity at different $z$-coordinates:

$$
\frac{\dot{\boldsymbol{u}}_{1 D}}{\dot{u}_{r}^{*}}=\frac{i \omega \boldsymbol{u}_{1 D}}{i \omega u_{r}^{*}}=\frac{\boldsymbol{u}_{1 D}}{u_{r}^{*}}=\boldsymbol{F}_{1}
$$

For the purposes of this contribution, it is necessary to define a second transfer function type, $\boldsymbol{F}_{2}$, which estimates the shear strain transfer functions w.r.t the velocity of the seismic excitation $\dot{u}_{r}^{*}$. This transfer function $\boldsymbol{F}_{2}$ is obtained from the relationship between shear strains and displacements:

$$
\gamma_{1 D}=\boldsymbol{F}_{\gamma \mathbf{u}}^{1 D} \boldsymbol{u}_{1 D}
$$


a)

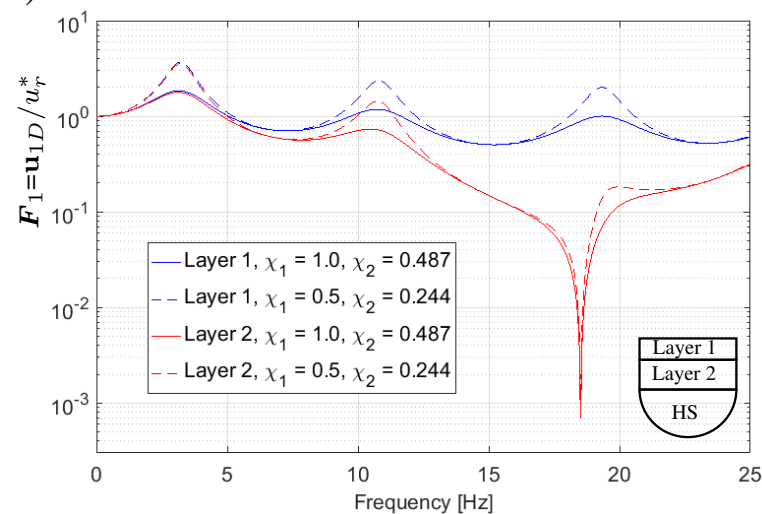

b)

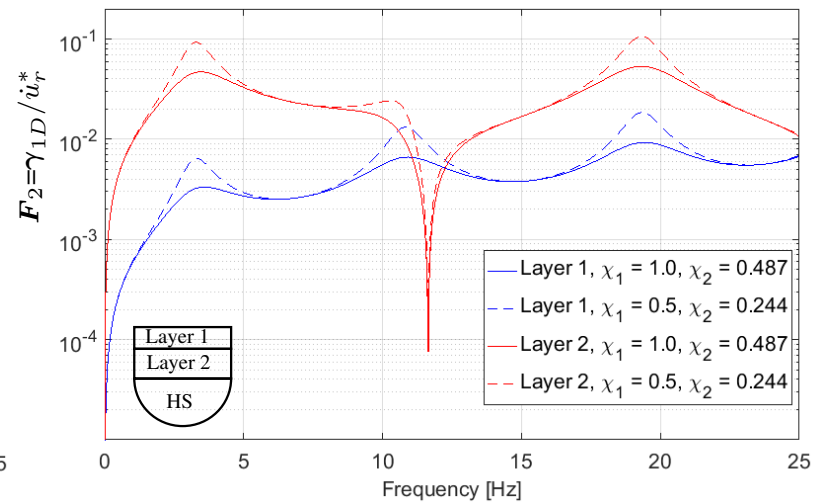

Figure 4: a) Displacement transfer function $\boldsymbol{F}_{1}$ and b) strain transfer functions $\boldsymbol{F}_{2}$, for the soils investigated in paragraph 2.3 .

where the matrix $\boldsymbol{F}_{\gamma \mathbf{u}}^{1 D}$ of the $N$-layered soil system reads:

$\boldsymbol{F}_{\gamma \mathbf{u}}^{1 D}=\left[\begin{array}{cccccc}-F_{1} & F_{12} & 0 & \ldots & 0 & 0 \\ -0.5 F_{12} & 0.5\left(F_{1}-F_{2}\right) & 0.5 F_{23} & 0 & \ldots & 0 \\ 0 & -0.5 F_{23} & 0.5\left(F_{2}-F_{3}\right) & 0.5 F_{34} & \ldots & 0 \\ \ldots & \ldots & \ldots & \ldots & \ldots & \ldots \\ 0 & \ldots & \ldots & -0.5 F_{N-1, N} & 0.5\left(F_{N-1}-F_{N}\right) & 0.5 F_{N, N+1} \\ 0 & \ldots & \ldots & 0 & -0.5 F_{N, N+1} & 0.5\left(F_{N}+F_{H S}\right)\end{array}\right]$

with

$$
\begin{aligned}
& n_{n}=\omega / c_{s, n} \\
& F_{n}=n_{n} \cot \left(n_{n} h_{n}\right) \text { and } F_{n, n+1}=n_{n} \sin ^{-1}\left(n_{n} h_{n}\right) \\
& F_{H S}=\frac{K_{H S}}{G_{H S}}=\frac{i \omega \rho_{H S} c_{s, H S}}{G_{H S}}
\end{aligned}
$$

Inserting Eq. (17) in Eq. (18), the functions $\boldsymbol{F}_{2}$ can be obtained from the functions $\boldsymbol{F}_{1}$ :

$$
\begin{aligned}
\gamma_{1 D} & =\boldsymbol{F}_{\gamma \mathbf{u}}^{1 D} \mathbf{u}_{1 D}=\boldsymbol{F}_{\gamma \mathbf{u}}^{1 D} \frac{1}{i \omega} \dot{\boldsymbol{u}}_{1 D}=\boldsymbol{F}_{\gamma \mathbf{u}}^{1 D} \frac{1}{i \omega} \boldsymbol{F}_{\mathbf{1}} \dot{u}_{r}^{*}=\boldsymbol{F}_{2} \dot{u}_{r}^{*} \\
\rightarrow \boldsymbol{F}_{2} & =\boldsymbol{\gamma} / \dot{u}_{r}^{*}=\boldsymbol{F}_{\gamma \mathbf{u}}^{1 D} \frac{1}{i \omega} \boldsymbol{F}_{1}
\end{aligned}
$$

The 1D transfer functions are a special case of the 3D relationships between input and output. It can be demonstrated that the 1D transfer functions $\boldsymbol{F}_{1}$ and $\boldsymbol{F}_{2}$ can be obtained from the 3D procedure described in paragraph 2.1 by setting $k_{x}=k_{y}=0$.

\subsection{Example: 1D Linear Transfer Function of a Layered Soil}

This example shows the transfer functions for a layered soil both in terms of $\boldsymbol{F}_{1}$ and $\boldsymbol{F}_{2}$. The investigation is carried out for two different soils, both made up of two undamped layers underlain by an elastic half space. The displacements transfer functions $\boldsymbol{F}_{1}$ are evaluated at the top surface of each layer, while the strain transfer functions $\boldsymbol{F}_{2}$ are evaluated at the center of each layer.

For both soils, the two layers have different thicknesses and shear velocities but same mass density: 
- Thickness $h_{1}=2.1336 \mathrm{~m}$ and $h_{2}=3.9624 \mathrm{~m}$

- Mass density $\rho_{1}=\rho_{2}=1922 \mathrm{~kg} / \mathrm{m}^{3}$

- Shear wave velocity $c_{s, 1}=158 \mathrm{~m} / \mathrm{s}$ and $c_{s, 2}=0.488 c_{s, 1}=77 \mathrm{~m} / \mathrm{s}$

The ratios $\chi_{i}$, which relate the impedance of the layers with the impedance of the half space, vary according to Tab. 1. Fig. 4 shows the linear transfer functions and the influence of the ratios $\chi_{i}$ on the amplification effect of the soil layering on the seismic input.

\begin{tabular}{c||c|c|}
\hline & $\chi_{1}=\frac{\rho_{1} c_{s, 1}}{\rho_{H S} c_{H S}}$ & $\chi_{2}=\frac{\rho_{2} c_{s, 2}}{\rho_{H S} c_{H S}}$ \\
\hline \hline Soil 1 (continuous lines in Fig. 4) & 1.0 & 0.488 \\
\hline Soil 2 (dashed lines in Fig. 4) & 0.5 & 0.244 \\
\hline
\end{tabular}

Table 1: Impedance ratios for the soils investigated in paragraph 2.3

\section{NONLINEAR SOIL BEHAVIOUR}

Experiments and past seismic events have shown that the soil material behaves non linearly when the strain exceeds $10^{-5}$, which is often the case during strong earthquakes [20, 3]. Adequate three-dimensional models for the description of the inelastic soil behavior are still under investigation today (for example in [15]). The salient characteristics of the behavior of the material is the degradation of the stiffness and the increasing dissipation with increasing strain. Fig. 5 shows typical degradation curves for the shear modulus $G$ normalized to the maximum shear modulus $G_{0}$ and for the hysteretic damping $\xi$ as a function of the cyclic strain $\gamma$ for sand material. The gray areas are the envelopes of experimental data according to [14], which show the influence of the confining pressure $p$ and of the void ratio $e$ on the degradation curves.

The most common models are based on the one-dimensional wave propagation theory and consider only one stress and one strain component at a time. Most of these models assume the behavior to be rate independent and fully characterized by the initial loading curve, or backbone. The most widely used stress-strain constitutive law is the Masing's rule [17]. This model assumes that unloading and reloading paths are scaled and/or inverted replicas of the initial stress-strain path for monotonic loading. In this model, when unloading or re-loading, the history of some or all of the reversal points must be tracked, so that when an unloading or re-loading episode intersects a previously taken path, that previous path is reestablished. Hence, this nonlinear model has memory of the previous strain history, but the path does not depend on the deformation speed. One can simulate any rate-independent, inelastic material obeying Masing's rule by means of a set of elasto-plastic springs in parallel [10]. The implementation of this model is much easier than the one for a non-linear backbone where many reversal points need to be tracked [7]. Other 1D models are the hyperbolic model [21] and the RambergOsgood [19, 16] model and more recent extensions of them, e.g. [5]. These assume that the stress-strain curve is independent of the cycle number, the void ratio, the confining pressure and the sand type.

In this contribution, we focus on the Assimaki et al. model [2], called MITS1, which is defined by 4 parameters and gives the strain-dependent values for the shear modulus and the hysteretic damping (or material damping) and takes into account the confining pressure. 

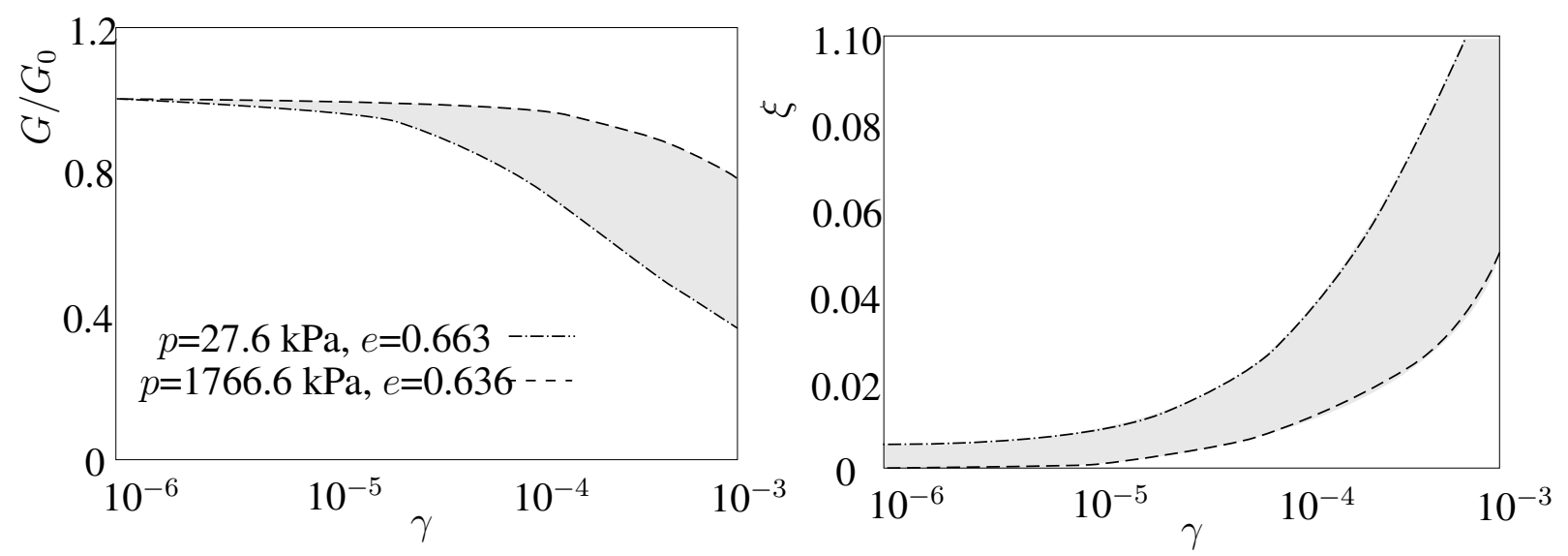

Figure 5: Experimental evidences of the dependency of the shear modulus $G$ and of the material damping $\xi$ on the strain $\gamma$, the confining pressure $p$ and on the void ratio $e$ (adapted from [2]).

Furthermore, to obtain a scalar measure of the shear deformation on a given point from the 3D strain vector, we use the octahedral shear strain [9]:

$$
\gamma_{\text {oct }}=\frac{2}{3} \sqrt{\left.\left(\varepsilon_{x}-\varepsilon_{y}\right)^{2}+\varepsilon_{y}-\varepsilon_{z}\right)^{2}+\left(\varepsilon_{z}-\varepsilon_{x}\right)^{2}+6 \gamma_{x y}^{2}+6 \gamma_{y z}^{2}+6 \gamma_{z x}^{2}}
$$

where $\varepsilon_{i}$ are the normal strains and $\gamma_{i}$ are the shear strains. This value is used as a reference for the degradation curves of the material model. It is important to notice that, if only shear strains in one direction would occur $\left(\varepsilon_{x}=\varepsilon_{y}=\varepsilon_{z}=\gamma_{x y}=\gamma_{y z}=0, \gamma_{z x} \neq 0\right)$, the octahedral shear strain $\gamma_{\text {oct }}$ does not coincide with the 1D shear strain $\gamma_{z x}\left(\gamma_{\text {oct }}=1.63 \gamma_{z x}\right)$.

\section{1D HFTD Method}

When a nonlinear constitutive material for the soil is assigned to the model, a time-integration algorithm is necessary, because the properties of the soil depends on the strain and the model must be updated at each time step. This task is computationally demanding and produces a large database of transient signal, which are difficult to interpret. An alternative to time integration schemes are the hybrid frequency-time domain (HFTD) methods, which assume a linearized material and solve the problem iteratively.

In the one dimensional case, the HFTD method involves the following steps:

1. discretize the soil in $N$ thin numerical layers;

2. assume initial linearized soil parameters;

3. evaluate the strain spectrum $\bar{\gamma}_{n}(\omega)$ at the center of each $n$-th numerical layer using the transfer functions $\boldsymbol{F}_{2}$, described earlier;

4. transform the response back into the time domain $\gamma_{n}(t)$ and evaluate the maximum of the signals;

5. for each layer, use this maximum to scale the strain spectrum, obtaining an effective strain spectrum $\bar{\gamma}_{n, \text { eff }}(\omega)$;

6. for each frequency $\omega$ of the spectrum, read the effective spectral strain value $\bar{\gamma}_{n \text {,eff }}(\omega)$ and use the constitutive laws (for example the hyperbolic model or the MITS1 model) to update the shear modulus $G_{n}\left(\bar{\gamma}_{n \text {,eff }}(\omega)\right)$ and the material damping $\xi_{n}\left(\bar{\gamma}_{n, \text { eff }}(\omega)\right)$; 
7. repeat the analysis until the maximum of the strain of two subsequent analysis converge.

With these procedure the frequency- and amplitude-dependent nature of the strains is approximated, which in turn requires the model's material to be frequency-dependent.

The effective strain spectra obtained in step 5 can be smoothed before using the strain value in the constitutive laws, in order to obtain a more stable iterative algorithm. The HFTD procedure presented by Kausel et al. [12] is summarized in Fig. 6

\subsection{Example: 1D HFTD Analysis of a Nonlinear Stratum}

As an example, a layer underlain by a bedrock (a stratum) is computed considering a linear and a nonlinear material. The soil properties are taken from [12]-Example 1. The total depth $h$ of the stratum is $25 \mathrm{~m}$, the density $\rho$ is $2000 \mathrm{~kg} / \mathrm{m}^{3}$, the shear velocity $c_{s} 200$ is $\mathrm{m} / \mathrm{s}$ and the material damping $\xi_{s}$ is $5 \%$. The nonlinear behavior follows the material model MITS1 [2]. The stratum is subjected to a transient signal of the Kobe Earthquake scaled to a maximum acceleration of $0.5 \mathrm{~g}$. The physical layer is discretized into 10 numerical layers and the exponential smoothing technique described in Fig. 6 is used.

Fig. 7 a shows the influence of the nonlinearity on the displacement transfer function $\boldsymbol{F}_{1}(\omega)$ evaluated at the surface. In the nonlinear case, the amplitudes of the peaks are decreased and the peaks for the first three resonances occur at lower frequencies then in the linear case. Fig. $7 \mathrm{~b}, \mathrm{c}$ and $\mathrm{d}$ show the spectrum of the strains at the center of the top layer, the transient accelerations and the transient displacements at the surface respectively, for the linear and non linear case.

Several alternative smoothing functions can be used to improve the method. Kwack et al. [13] compared different smoothing techniques found in literature. Wang and Zhao [22] proposed a smoothing method based on a moving average method and a different scaling technique for the computation of the effective strain. Fig. 8 shows the influence of the different smoothing techniques for the effective strain (Fig. $8 \mathrm{~d}$ ) on the converged results, e.g. on the accelerations of the free field at the surface (Fig. 8a) and on the displacement transfer functions (Fig. 8p). The moving average method (MAM) gives the most stable and realistic results.

Fig. $8 \mathrm{c}$ shows the frequency-dependent behavior of the material parameters for the MITS 1 model using different smoothing techniques, evaluated at the center of the physical layer. It can be observed that the shear modulus is strongly degraded at low frequencies. The opposite applies to the hysteric damping, which decreases strongly at high frequencies. This is also confirmed in Fig. 9, where the distribution of the material properties with depth is shown for different frequencies, for the converged solution.

Fig 10 shows a validation of the 1D HFTD method against a fully transient nonlinear analysis carried out with the aid of a series of elasto-plastic springs, according to the Ivan's Model [11 $]^{2}$, The comparison shows the great agreement between the two approaches, confirming the suitability of the hybrid method.

\section{3D HFTD Method}

All the existing studies on HFTD methods for the SSI simulations neglect the effects of the three dimensions of the system and focus only on one strain component at time. Therefore, we propose to extend the HFTD methods by computing the three dimensional strains for the free field and iteratively solve the problem analogously to the 1D procedure. Fig. 11 shows the proposed algorithm for the 3D HFTD methods. The main difference between the $1 \mathrm{D}$ and the

\footnotetext{
${ }^{2}$ Pages 555
} 


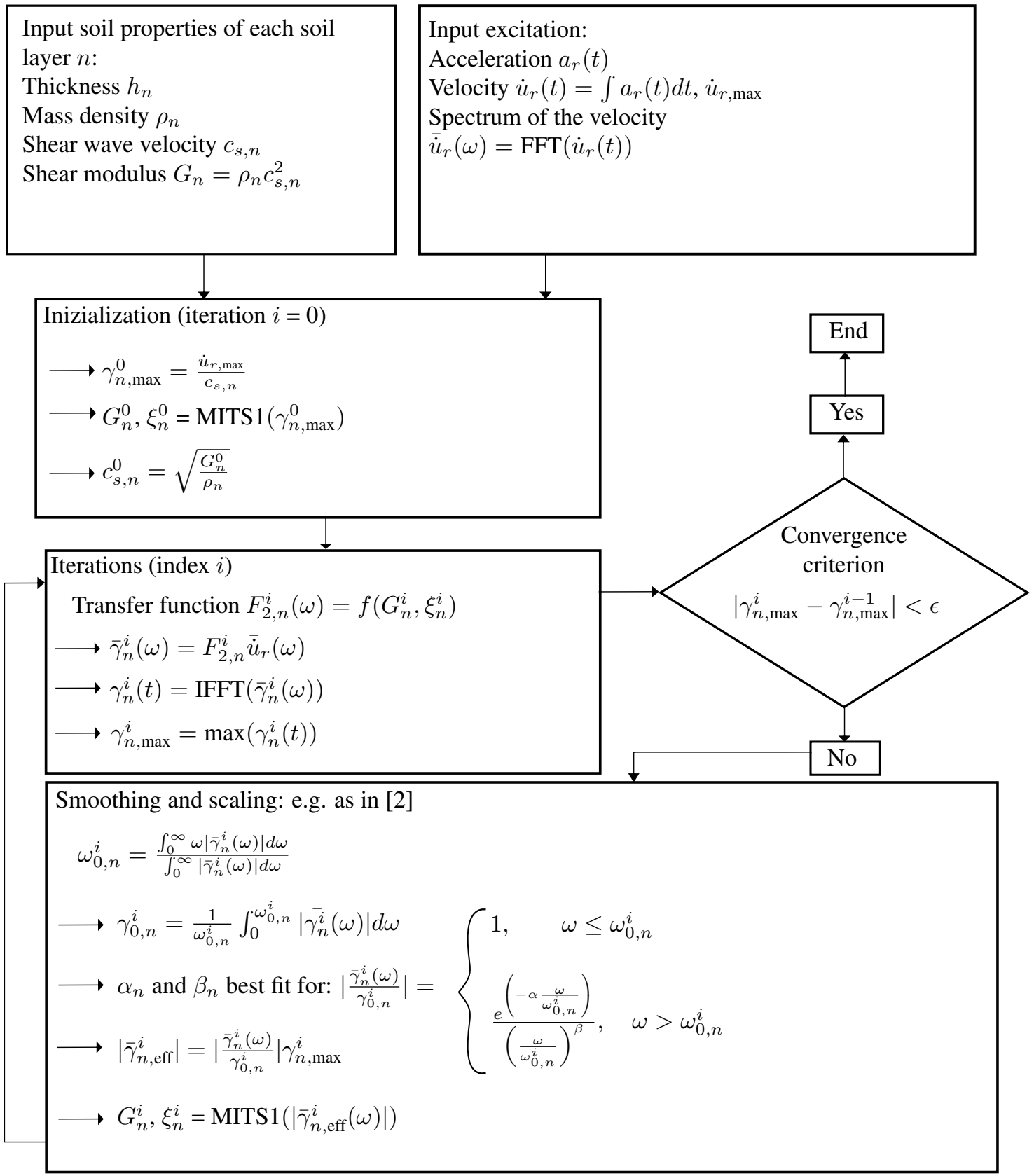

Figure 6: 1D iterative process according to [2] for non-linear soil properties based on the MITS1 constitutive laws. 


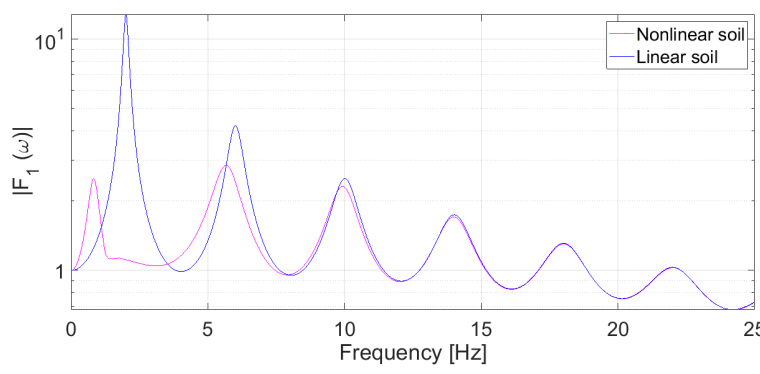

(a) Transfer function $\boldsymbol{F}_{1}$ at the surface

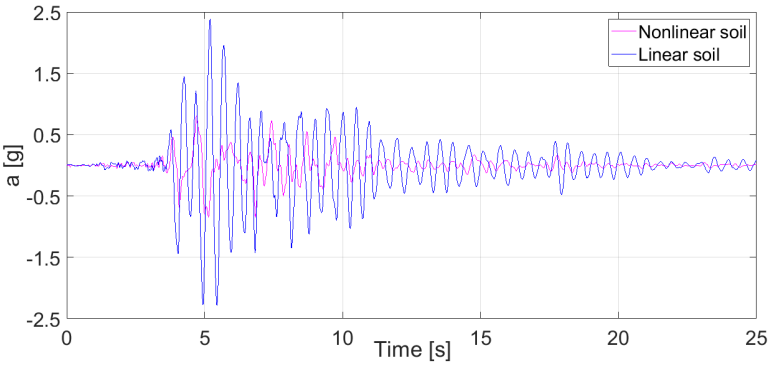

(c) Acceleration response at the surface

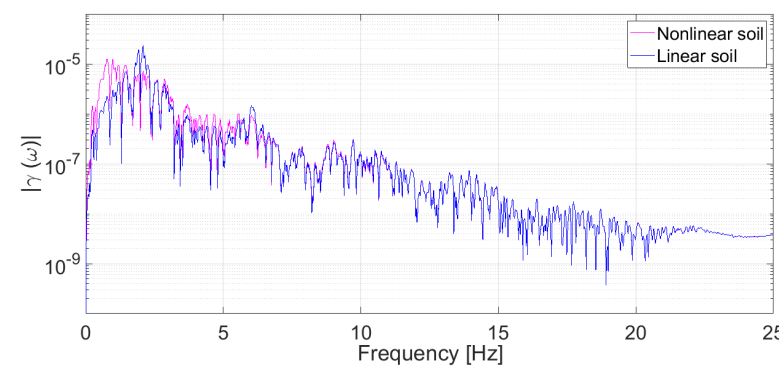

(b) Strain spectrum at center of the numerical layer on top

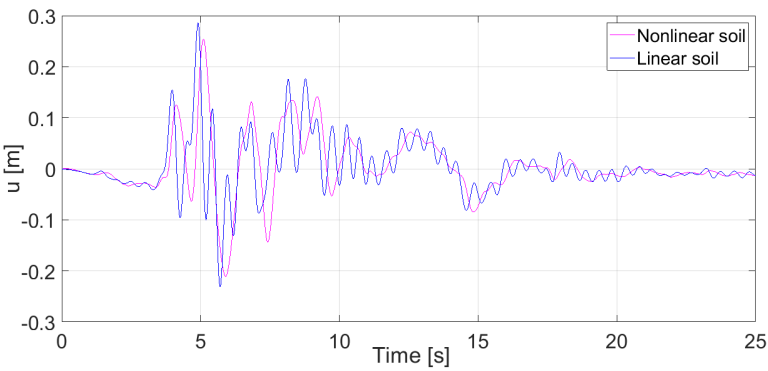

(d) Displacement response at the surface

Figure 7: Comparison between the nonlinear soil response resulting from the 1D HFTD method and the linear soil response.

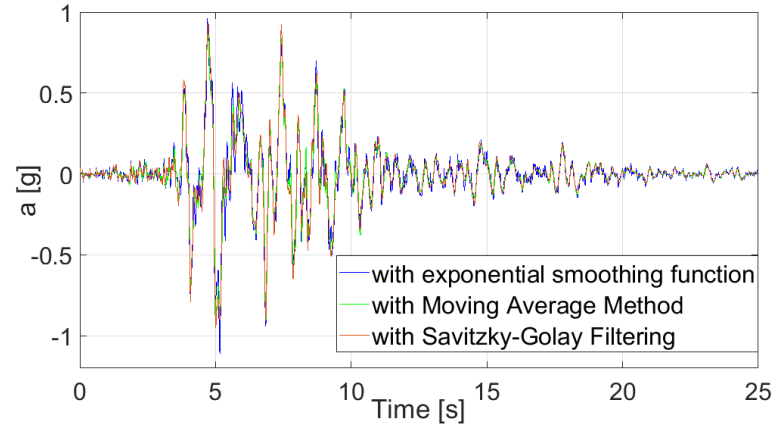

(a) Acceleration at surface

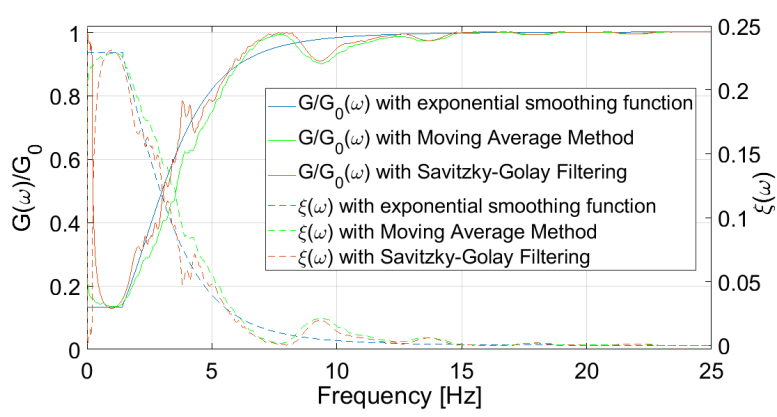

(c) Degradation curves at center of the physical layer

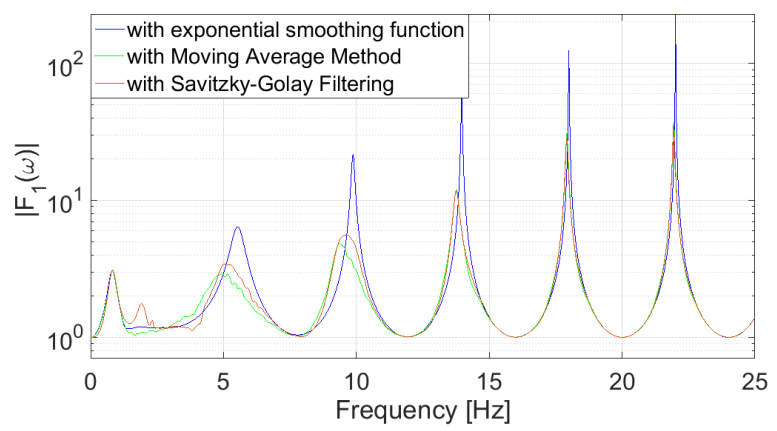

(b) Transfer function $F_{1}$

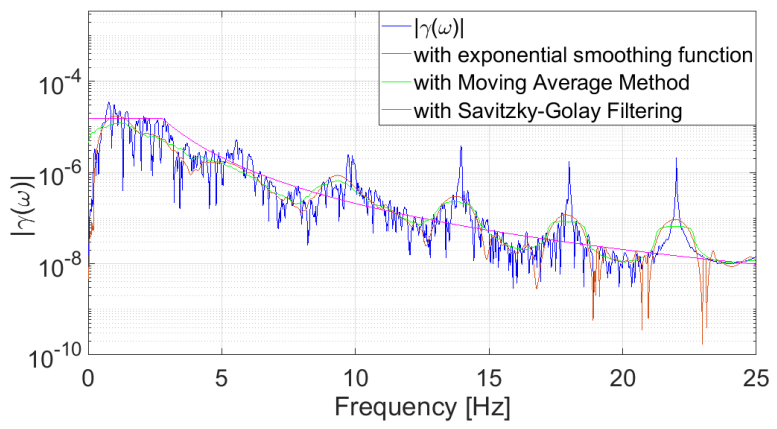

(d) Strain spectrum at center of the upper numerical layer

Figure 8: Nonlinear soil response with different smoothing techniques for the 1D case. 

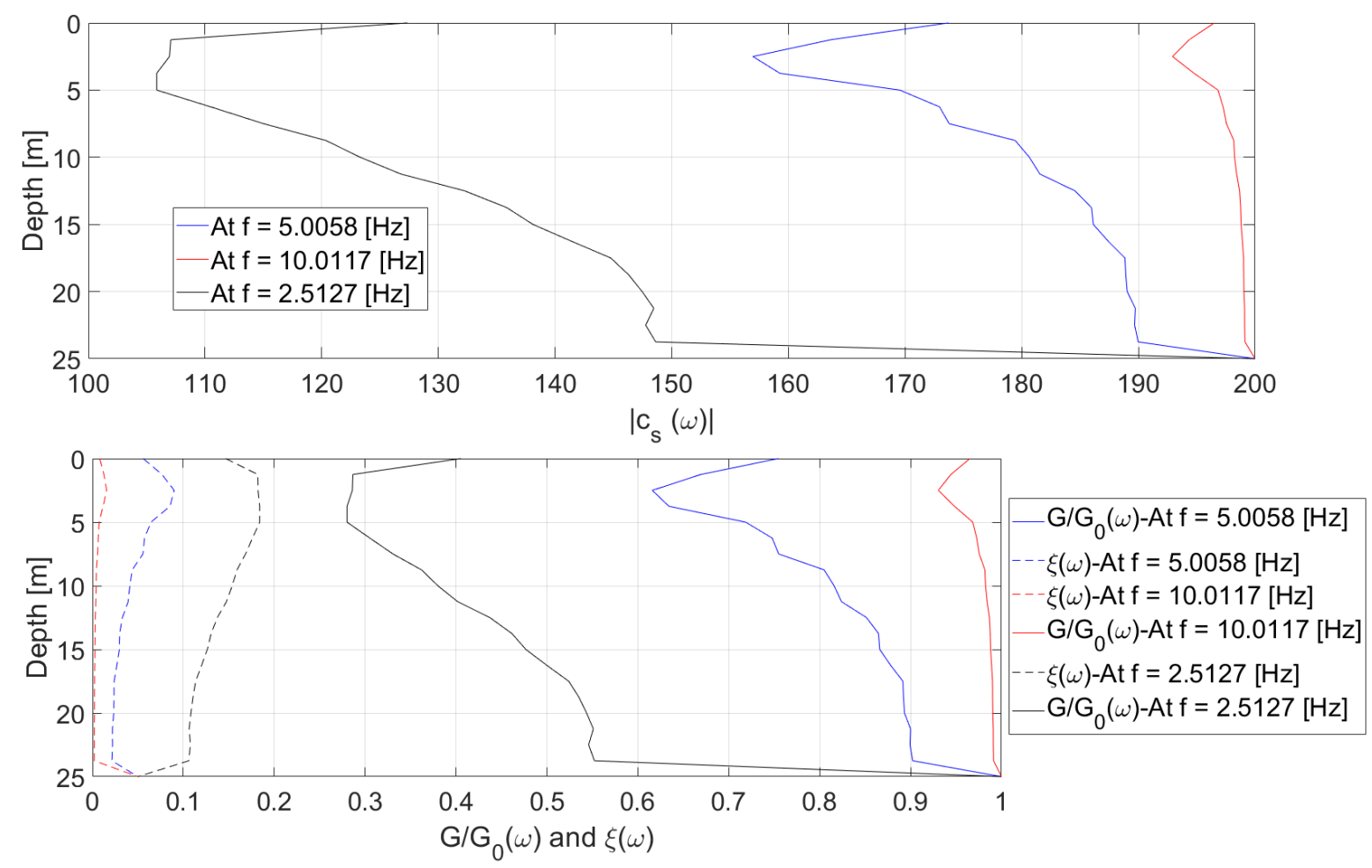

Figure 9: Nonlinear soil properties over depth at frequency of $2.5 \mathrm{~Hz}, 5 \mathrm{~Hz}$ and $10 \mathrm{~Hz}$.

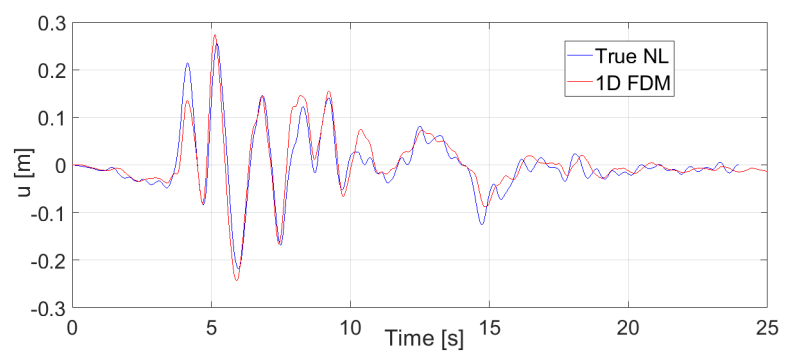

(a) Displacement on the soil surface

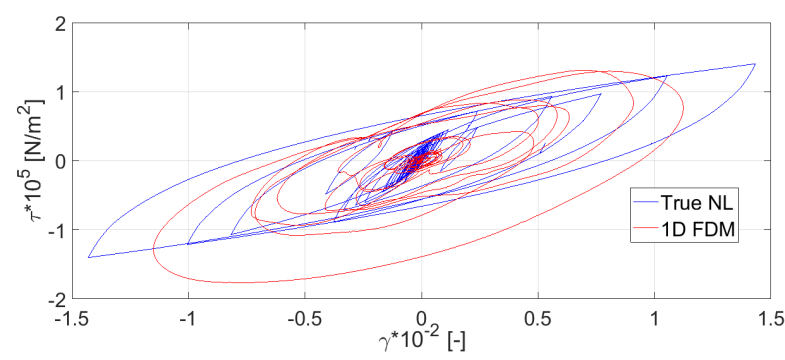

(c) Comparison of stress-strain curve

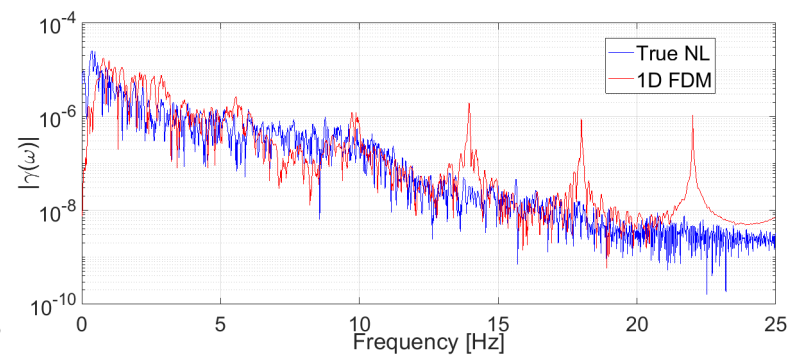

(b) Strain spectrum - center of the numerical top layer

Figure 10: Comparison between the results according to the 1D HFTD method and a true nonlinear simulation using elasto-plastic springs after Ivan [11]. 
3D lies on the computation of the strains: in the 1D case, the strains at different depths in the free field are obtained through the 1D transfer functions $\boldsymbol{F}_{2}$ according to Eq. 21); in the 3D case, the strains are obtained with the ITM according to Eq. (13).

In the 3D HFTD method, it is possible to consider a complex spatial distribution of the input at the rock outcrop or at any point of the soil. Consequently, also the displacements and strains become a function of the coordinates $x, y$ and $z$. These can be used to update the soil properties locally, thus obtaining a non-homogenous anisotropic soil. However, one of the assumption of the ITM method is that the soil has the same properties in $x$-direction and $y$-direction and that the properties in $z$-direction are homogenous within each horizontal layer. Therefore, for the iterative part of the method, we approximate the 3D strain distribution through a distribution which is constant for each horizontal layer and the reference strain vector $\varepsilon_{n}$ for each layer $n$ corresponds to the octahedral shear strain evaluated at the coordinate $z_{n}$ (depth of the center of each layer), $\widehat{x}$ and $\widehat{y}$ (horizontal coordinate of the evaluation points, e.g. directly underneath the potential structure coupled to the free field). With the components of the reference strain vector, the octahedral shear strain according to Eq. (22) is computed and used to update the material properties of each layer with the chosen nonlinear material law. The more horizontal numerical layers are considered, the more accurate is the approximation. Alternatively one can used directly the 6 components of the the reference strain vector $\varepsilon_{n}$ and a 3D nonlinear material model to update the soil model.

\subsection{Comparison Between the 1D and 3D HFTD Method}

In order to compare the $1 \mathrm{D}$ and 3D results, a free field subjected to a plane S-wave is considered. The wave propagates vertically while the particles move horizontally in $\mathrm{x}$-direction $\left(v_{r}^{*}=w_{r}^{*}=0, u_{r}^{*} \neq 0\right)$ according to the Kobe earthquake signal[12]. In each plane $x-y$, all the particles experience the same horizontal motion, therefore the three dimensional case coincide with a one-dimensional problem. The soil is made up of a physical layer underlain by an elastic half space and the properties are specified in Tab. 2. The physical layer is divided into 10 numerical layers. The evaluation points for the ITM quantities are at the coordinate $x_{n}=y_{n}=0$ and at the $z_{n}$ corresponding to the depths of the center of the $N$ numerical layers.

For the solution of the ITM, the following boundary conditions are applied:

- BC 1: zero stresses at the soil surface;

- BC 2: the product of the dynamic stiffness of the half space with the relative displacements at the lowest interface of the layer is equal to the stresses at this interface,

$$
\begin{aligned}
& {\left[\bar{\sigma}_{z x}(z=h), \bar{\sigma}_{y z}(z=h), \bar{\sigma}_{z}\left(z_{1}=h\right)\right]^{T}=} \\
& \mathbf{K}_{H S}\left[\left(\bar{u}(z=h)-\bar{u}_{r}^{*}\right),\left(\bar{v}(z=h)-\bar{v}_{r}^{*}\right),\left(\bar{w}(z=h)-\bar{w}_{r}^{*}\right)\right]^{T} ;
\end{aligned}
$$

- BC 3: continuity at interfaces between layers.

Where $h$ is the thickness of the physical soil layer. By applying these boundary conditions, the coefficient vector $c$ can be found and consequently the free field soil response in the image space $\left(k_{x}, k_{y}, z, \omega\right)$ is obtained. The solution in the original space $(x, y, z, t)$ is computed through the inverse Fourier transformation.

The strain at the layer center and the acceleration at the surface resulting from 1D (blue curves) and 3D-model (red curves) are plotted in Fig. 12a and c. It can be seen that strains and accelerations resulting from both models is nearly identical. The 3D model achieved the convergence after eight iterations while the $1 \mathrm{D}$ model required six iteration steps. Also the 
Input soil properties of each soil layer $n$ :

Thickness $h_{n}$

Mass density $\rho_{n}$

Shear wave velocity $c_{s, n}$

Shear modulus $G_{n}=\rho_{n} c_{s, n}^{2}$

(isotropic material: $G_{n, x}=G_{n, y}=G_{n}$ )
Input excitation:

Acceleration $\boldsymbol{a}_{r}^{*}(x, y, z, t)$

Velocity $\dot{\boldsymbol{u}}_{r}^{*}(x, y, z, t)(t)=\int \boldsymbol{a}_{r}^{*}(t) d t, \dot{\boldsymbol{u}}_{r, \max }^{*}$

(bold symbols $\boldsymbol{a}_{r}^{*}$ and $\dot{\boldsymbol{u}}_{r}^{*}$ are vectors containing components

in $\mathrm{x}, \mathrm{y}$ and $\mathrm{z}$ direction)

$$
\begin{aligned}
& \text { Inizialization (iteration } i=0, \mathrm{j}=\mathrm{x}, \mathrm{y}) \\
& \longrightarrow \gamma_{n, \max }^{0}=\max \left(\frac{\dot{u}_{r, \max }^{*}}{c_{s, n}}, \frac{\dot{v}_{r, \max }^{*}}{c_{s, n}}\right) \\
& \longrightarrow G_{n}^{0}, \xi_{n}^{0}=\operatorname{MITS} 1\left(\gamma_{n, \max }^{0}\right) \\
& \longrightarrow c_{s, n}^{0}=\sqrt{\frac{G_{n}^{0}}{\rho_{n}}}
\end{aligned}
$$

Iterations (index $i$ )

Using ITM

$\longrightarrow \bar{\varepsilon}^{i}(\omega, x, y, z)$

For $z_{n}$ equal to the depth of the center of the $n$-th layer

and $\widehat{x}$ and $\widehat{y}$ equal to the coordinates of the evaluation point

in the $n$-th horizontal plane

$\longrightarrow \bar{\varepsilon}_{n}^{i}(\omega)=\bar{\varepsilon}^{i}\left(\omega, \widehat{x}, \widehat{y}, z_{n}\right)$
$\longrightarrow \gamma_{n, \text { oct }}^{i}(\omega)$ after Eq. 22
$\longrightarrow \gamma_{n, \max }^{i}=\max \left(\operatorname{IFFT}\left(\gamma_{n, \text { oct }}^{i}(\omega)\right)\right)$

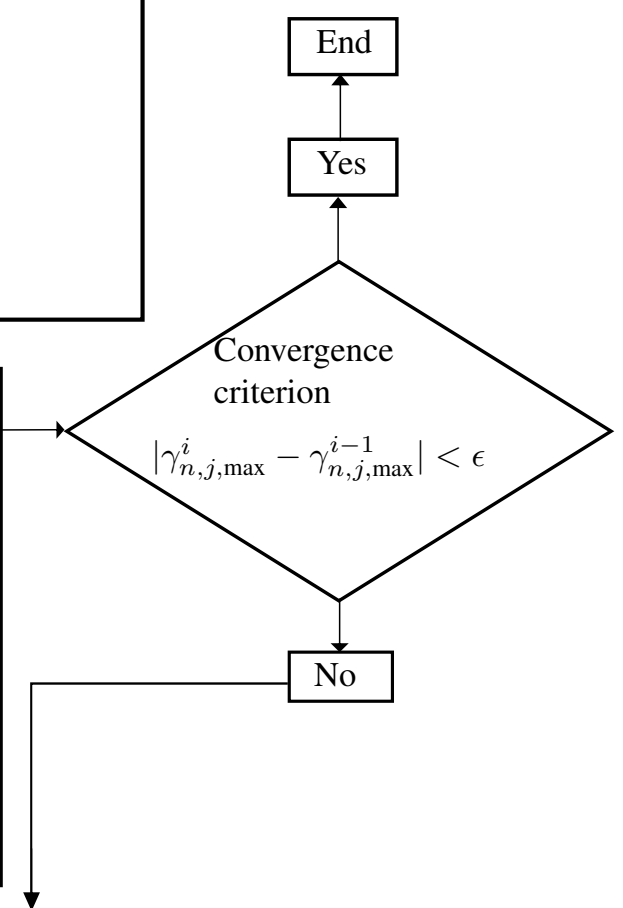

Smoothind and scaling

$\longrightarrow\left|\bar{\gamma}_{n, \mathrm{eff}}^{i}\right|=\left|\bar{\gamma}_{n, \text { smooth }}^{i}(\omega)\right| \gamma_{n, \max }^{i}$

$\longrightarrow G_{n}^{i}, \xi_{n}^{i}=\operatorname{MITS} 1\left(\left|\bar{\gamma}_{n, \mathrm{eff}}^{i}\right|\right)$

$\longrightarrow E_{n}^{i}$ and $c_{p, n}^{i}$ are calculated with new values of $G_{n}^{i}, \xi_{n}^{i}$

Figure 11: Proposed 3D iterative process for non-linear soil properties based on the MITS1 constitutive laws.

\begin{tabular}{l||l|l|l|l|l} 
& $h[\mathrm{~m}]$ & $\rho\left[\mathrm{kg} / \mathrm{m}^{3}\right]$ & $c_{s}[\mathrm{~m} / \mathrm{s}]$ & $\xi[-]$ & $\nu[-]$ \\
\hline \hline Layer & 6.096 & 2002 & 244 & 0.05 & 0.3 \\
\hline Half space (HS) & - & 2102 & 265 & 0.05 & 0.3
\end{tabular}

Table 2: Soil properties - Example with ITM 


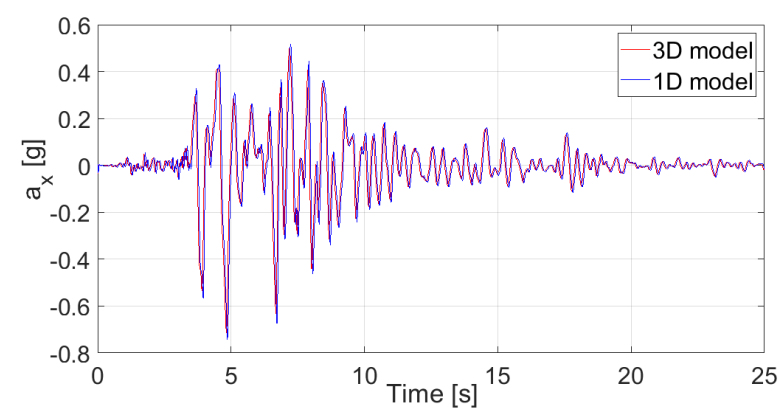

(a) Acceleration in $\mathrm{x}$ direction at surface

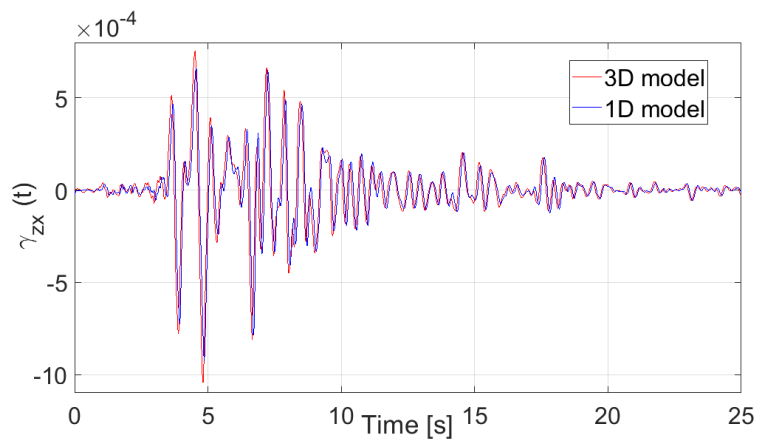

(c) Shear strain in $\mathrm{x}$ direction $\gamma_{z x}(t)$ at layer center

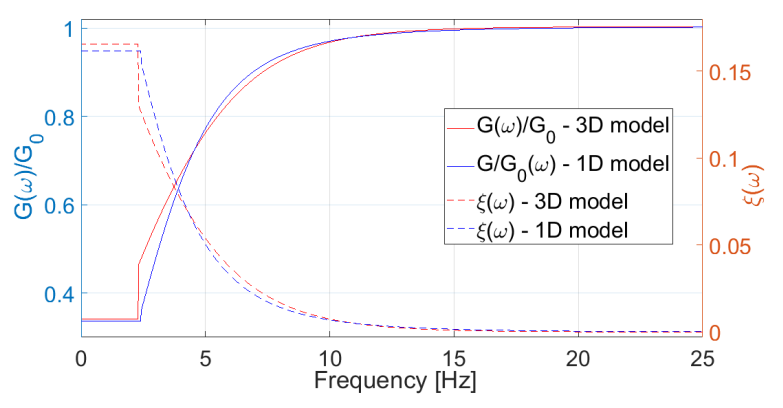

(b) Degradation curve at layer center

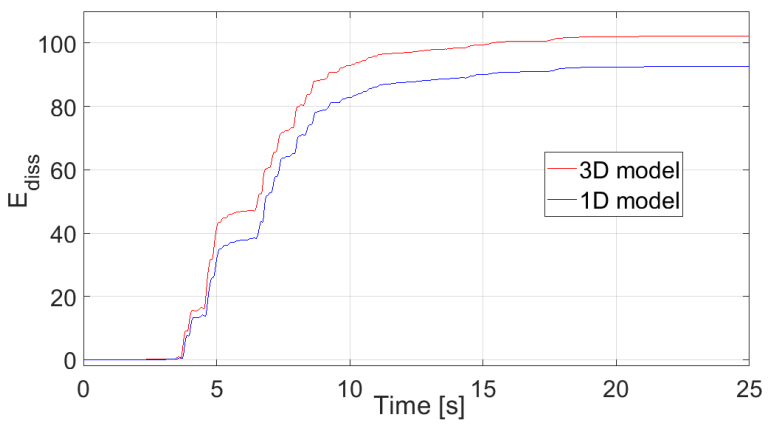

(d) Dissipated energy at layer center

Figure 12: Nonlinear response of one heterogeneous layer on elastic half space, subjected to a plane wave: 3DHFTD method with ITM versus 1D-HFTD method.

degradation curves and the dissipated energy shows agrrement between the $1 \mathrm{D}$ and the $3 \mathrm{D}$ method.

\subsection{D HFTD Method Considering a Spatially Varying Seismic Excitation}

In this example a layered soil is subjected to a seismic excitation with a non-constant horizontal distribution, applied at the interface between layer and half space, as shown in Fig 13 . The material properties are shown in Tab. 3. Fig. 14 shows the transient signals and the spectra of the input $u_{r}^{*}$ in $x$-direction and of $v_{r}^{*}$ in $y$-direction, applied to all the particles of the area highlighted in Fig 14. The loaded area is $0.625 \mathrm{~m} \times 0.625 \mathrm{~m}$, symmetric with respect to the point $(x, y)=(0,0)$. Because of the small size of the loaded area, the excitation which would results from this excitation would be very small. Therefore, the equivalent seismic loads $\left[\bar{\sigma}_{z x}(z=h), \bar{\sigma}_{y z}(z=h), \bar{\sigma}_{z}\left(z_{1}=h\right)\right]^{T}$ are scaled with a factor of 1200 in order to introduced enough energy into the system. This scenario does not represents a real seismic loading condition but serves the purpose of showing the effect of a localized excitation. The soil is discretized into 10 numerical layers and the MITS1 material model is assumed. The reference strains for the iterative process are evaluated at the center of each layer and the the points $(x, y)=(0,0)$. The results of this scenario are compared to the results of the same scenario considering a linear material behavior.

Fig. 15 shows the time history of the displacement response of the soil surface at $(x, y, z)=$ $(0,0,0)$, and the strain response at a depth of $5 \mathrm{~m}$ (center of the physical layer), for the nonlinear and linear case. Fig. 16 shows the contour of the displacement response in $x$ - and $y$-direction over the considered area on the soil surface at a chosen time $t=5.5 \mathrm{~s}$. Finally, Fig. 17 shows the transfer functions $F_{1, x}=u / u_{r}^{*}$ and $F_{1, y}=v / v_{r}^{*}$ (defined as in the $1 \mathrm{D}$ case) as well as the spectrum of the total displacements $u_{t o t}(\omega, x=y=z=0)=\sqrt{u^{2}+v^{2}+w^{2}}$ at the soil 


\begin{tabular}{c||c|c|c|c|c|}
\hline & $h[\mathrm{~m}]$ & $\rho\left[\mathrm{kg} / \mathrm{m}^{3}\right]$ & $c_{s}[\mathrm{~m} / \mathrm{s}]$ & $\xi[-]$ & $\nu[-]$ \\
\hline \hline Layer & 10.0 & 2002 & 100 & 0.05 & 0.3 \\
\hline Half space & - & 2102 & 265 & 0.05 & 0.3 \\
\hline
\end{tabular}

Table 3: Soil properties for the 3D investigation in paragraph 5.2

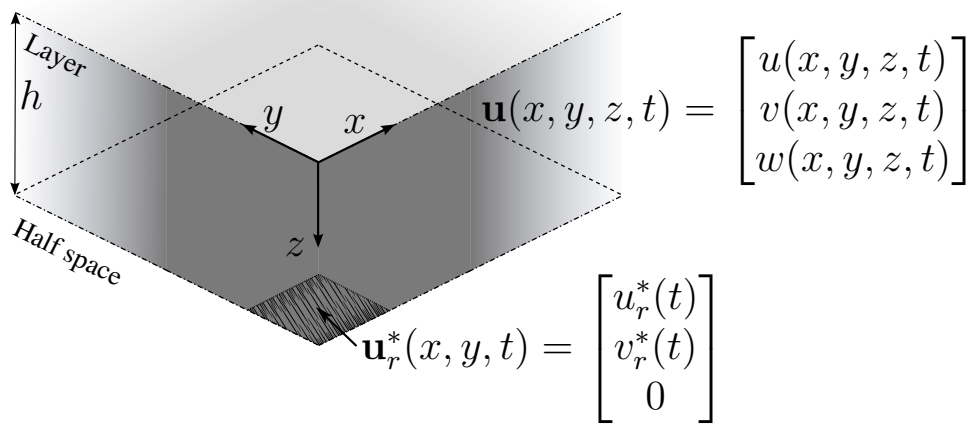

Figure 13: Non-constant spatial distribution of the seismic excitation at the layer-HS interface for the example in paragraph 5.2 .

surface for the linear and the nonlinear case. The results show the influence of the nonlinear behavior on the frequency response and on the transient response.

\subsection{Limitations of the 3D HFTD Method}

The proposed method owns several advantages with respect to fully transient nonlinear analysis such as efficiency, simplicity of application and recued amount of output data. However, several limitations are also present and discussed in the following:

- Linearization: as in the 1D HFTD method, we assume that the transient nonlinear behavior of the 3D ground can be approximated by a superposition of steady-state linearized frequency-dependent responses. The convergence criteria focuses on the strain and affect the accuracy of the iterative process. This assumption needs to be investigated in more details, with a comparison against a fully transient nonlinear analysis.

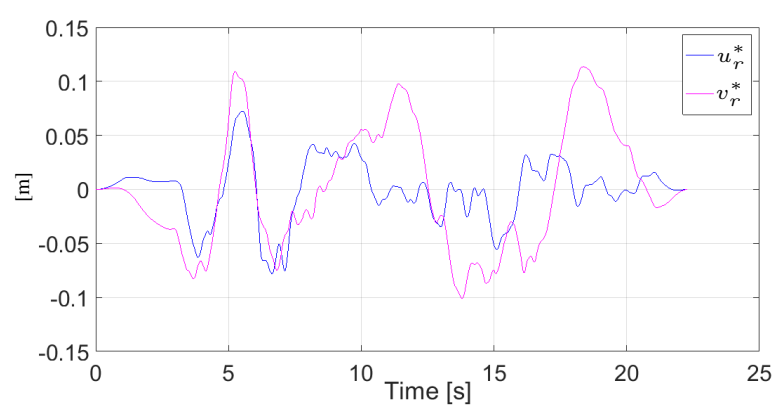

(a) Transient signals of the input motion (b) Spectra of the input motion

Figure 14: Displacement input in $x$ and $y$ direction applied at a portion of the layer-HS interface - 1987 earthquake in Superstition Hills, California, USA (downloaded from the PEER Strong Motion Database Record [1]). 


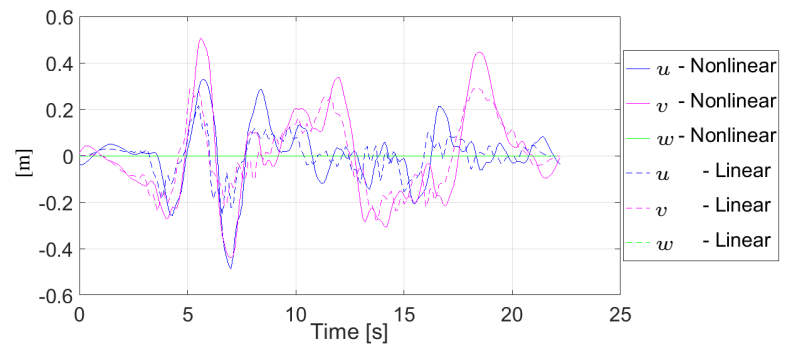

(a) Displacements at the soil surface (b) Strains at $z=5 \mathrm{~m}$

Figure 15: Comparison of the displacement and strain response at $(x, y)=(0,0)$ for the linear and the nonlinear 3D HFTD method.

(a) Contour plot of the displacement $u(x, y, z=0, t=5.5 s)$ (b) Contour plot of the displacement $v(x, y, z=0, t=5.5 s)$

Figure 16: Contour of the displacement response of the soil surface at $t=5.5 \mathrm{~s}$ for the nonlinear case.

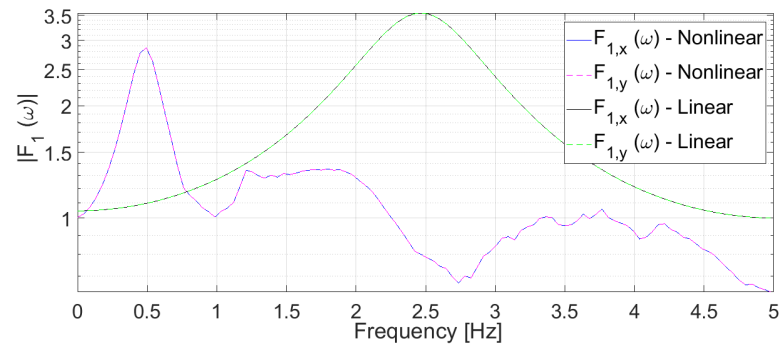

(a) Transfer functions of the displacement response on the soil surface

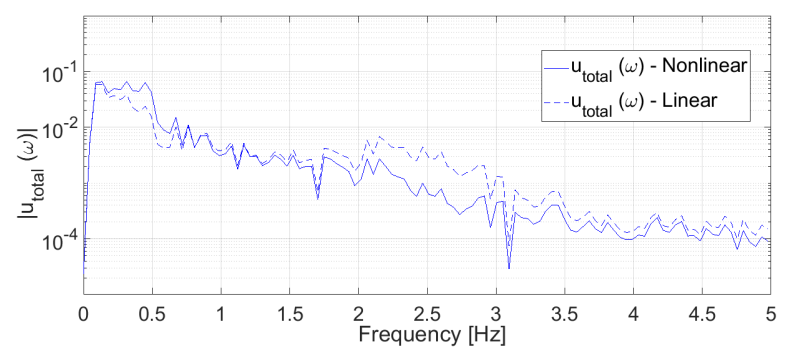

(b) Spectrum of the total displacement response on the soil surface

Figure 17: Comparison of the transfer functions and total displacement spectrum at the soil surface at $(x, y)=(0$, $0)$ - Linear versus nonlinear soil system. 
- Material model: most of the nonlinear material model for soils are one-dimansional and the material parameters are defines as a function of the shear deformation. In the 3D HFTD method, one obtains a more detailed picture of strain deformation with 6 components, which could be the input of a realistic three-dimensional model for the description of the inelastic soil behavior. However, 3D material models are still under investigation. Therefore, we opted for established 1D material models, such as the MITS1, which depend only on one component of the strain. Here, we take the octahedral shear strain as a scalar measure of the shear deformation on a given point. This aspect can be improved integrating more complex 3D material models.

- Spatial averaging: in the 1D HFTD method, the system is approximated by a one dimensional model and all the particles in the $x$-y-planes, perpendicular to the depth-direction ( $z$-axis), moves in phase and with the same amplitude. In the 3D HFTD method, the distribution of the motions and strains in the $x$-y-planes is not constant, being the input arbitrarily spatially distributed. One could use this information to update the soil model locally. However, in the current formulation of the ITM, we assume that each horizontal layer is isotropic. Therefore, the material properties, such as $G$ and $\xi$, are constant for the whole $x$-z-plane and over the whole depth of the layer. For this reason, we have to summarize the spatial distribution into one reference value, for example observing one specific horizontal point $(\widehat{x}, \widehat{y})$ to obtain the reference strain for the material model. In future developments, we plan to reformulate the method including spatial averaging based on energy consideration and to extend the ITM solution for anisotropic and locally inhomogeneous soils.

\section{CONCLUSIONS}

In this contribution we gave an overview of the one-dimensional (1D) hybrid frequency-time domain (HFTD) method for the simulation of the seismic response of nonlinear soils. The 1D HFTD method does not takes into account the three dimensional (3D) effects that characterize the wave propagation in the soil. To fill this gap, we presented a 3D HFTD method, where the seismic input motions can be applied at any point of the soil and can be spatially variable. The effective strains in the soil are computed based on the 3D theory of elasticity through the integral transform method (ITM). The 3D strain vector is used to find the linearized properties of the nonlinear soil and the problem is solved iteratively transforming from the image space into the original space and viceversa and updating the soil properties until convergence. Several examples show the comparison between the 1D and the 3D formulations and between linear and nonlinear simulations.

In the proposed method the main assumptions are that the soil is isotropic in the horizontal plane and that the properties of the material are homogenous within each horizontal layer. Moreover, the nonlinear material model used to update the materiel properties is one-dimensional. In future works, the 3D HFTD method will be extended for anisotropic soils and for three dimensional non linear material models.

\section{REFERENCES}

[1] Peer strong motion database record. https://ngawest2.berkeley.edu/. Accessed: 2019-01-15. 
[2] Dominic Assimaki, Eduardo Kausel, and Andrew Whittle. Model for dynamic shear modulus and damping for granular soils. J Geotech Geoenviron, 126(10):859-869, 2000.

[3] Igor A Beresnev and Kuo-Liang Wen. Nonlinear soil response-a reality? Bulletin of the Seismological Society of America, 86(6):1964-1978, 1996.

[4] Julian Freisinger and Gerhard Müller. Modellierung eines halbraums mit sphärischem oder zylinderförmigem hohlraum für dreidimensionale boden-bauwerk-interaktion. Forschung im Ingenieurwesen, pages 1-12, 2018.

[5] David R Groholski, Youssef MA Hashash, Byungmin Kim, Michael Musgrove, Joseph Harmon, and Jonathan P Stewart. Simplified model for small-strain nonlinearity and strength in 1d seismic site response analysis. Journal of Geotechnical and Geoenvironmental Engineering, 142(9):04016042, 2016.

[6] Manuela Hackenberg and Gerhard Müller. Modeling a halfspace with tunnel using a coupled integral transform method-finite element method approach. PAMM, 15(1):389$390,2015$.

[7] Youssef MA Hashash and Duhee Park. Non-linear one-dimensional seismic ground motion propagation in the mississippi embayment. Eng Geol, 62(1):185-206, 2001.

[8] George D Hatzigeorgiou and Dimitri E Beskos. Soil-structure interaction effects on seismic inelastic analysis of 3-d tunnels. Soil Dynamics and Earthquake Engineering, 30(9):851-861, 2010.

[9] Chitas P.; Santos J.A.; Lopes I.F. A three-dimensional extension of the ramberg-osgood model. comparison with other formulations. In Proceedings of the 15WCEE, 2012.

[10] Wilfred D Iwan. On a class of models for the yielding behavior of continuous and composite systems. J Appl Mech, 1967.

[11] Eduardo Kausel. Advanced structural dynamics. Cambridge University Press, 2017.

[12] Eduardo Kausel and Dominic Assimaki. Seismic simulation of inelastic soils via frequency-dependent moduli and damping. Journal of Engineering Mechanics, 128(1):34-47, 2002.

[13] Dong-Yeop Kwak, Chang-Gyun Jeong, Duhee Park, and Sisam Park. Comparison of frequency dependent equivalent linear analysis methods. In The 14th World Conference on Earthquake Engineering, page 8, 2008.

[14] JP Laird and KH Stokoe. Dynamic properties of remolded and undisturbed soil samples tested at high confining pressures. Geotechnical Engineering Report GR93, 6, 1993.

[15] Chao Ma, Dechun Lu, Xiuli Du, and Annan Zhou. Developing a 3d elastoplastic constitutive model for soils: A new approach based on characteristic stress. Computers and Geotechnics, 86:129-140, 2017.

[16] Luan Maotian. Ramberg-osgood constitutive model with variable parameters for dynamic nonlinear analysis of soils. Earthq Eng Eng Vib, 2:007, 1992. 
[17] Georg MASING. Eigenspannungen und verfestigung beim messing. In Proceedings, second international congress of applied mechanics, pages 332-335, 1926.

[18] Hossein Rahnema, Sassan Mohasseb, and Behtash JavidSharifi. 2-d soil-structure interaction in time domain by the sbfem and two non-linear soil models. Soil Dynamics and Earthquake Engineering, 88:152-175, 2016.

[19] Walter Ramberg and William R Osgood. Description of stress-strain curves by three parameters. National advisory committee for aeronautics, 1943.

[20] Julie Régnier, Héloïse Cadet, and Pierre-Yves Bard. Empirical quantification of the impact of nonlinear soil behavior on site response. Bulletin of the Seismological Society of America, 106(4):1710-1719, 2016.

[21] H Bolton Seed, Robert T Wong, IM Idriss, and Kohji Tokimatsu. Moduli and damping factors for dynamic analyses of cohesionless soils. J Geotech Eng, 112(11):1016-1032, 1986.

[22] Duguo Wang and Chenggang Zhao. Strain-threshold-and frequency-dependent seismic simulation of nonlinear soils. Earthquake Science, 27(6):615-626, 2014.

[23] J Wolf and William Hall. Soil-structure-interaction analysis in time domain. Number LCH-BOOK-2008-037. A Division of Simon \& Schuster, 1988. 